\title{
Criterion for Generalized Weakly Fuzzy Invex Monotonocities
}

\author{
Meraj A. Khan $\left(\mathbb{D},{ }^{1}\right.$ Izhar Ahmad $\left(\mathbb{D},{ }^{2}\right.$ and Abdulrahman Aljohani ${ }^{1}$ \\ ${ }^{1}$ Department of Mathematics, University of Tabuk, Tabuk, Saudi Arabia \\ ${ }^{2}$ Department of Mathematics and Statistics, King Fahd University of Petroleum and Minerals, Dhahran 31261, Saudi Arabia \\ Correspondence should be addressed to Meraj A. Khan; meraj79@gmail.com
}

Received 30 November 2017; Accepted 7 March 2018; Published 11 April 2018

Academic Editor: Pushpinder Singh

Copyright (C) 2018 Meraj A. Khan et al. This is an open access article distributed under the Creative Commons Attribution License, which permits unrestricted use, distribution, and reproduction in any medium, provided the original work is properly cited.

\begin{abstract}
The present paper deals with the concepts of generalized fuzzy invex monotonocities and generalized weakly fuzzy invex functions. Some necessary conditions for weakly fuzzy invex monotonocities are presented. Moreover, the concept of fuzzy strong invex monotonocities and fuzzy strong invex functions are also discussed. To strengthen our definitions, we provide nontrivial examples of fuzzy invex monotonocities and weakly fuzzy invex functions.
\end{abstract}

\section{Introduction}

In the last few years, the conception of convexity and generalized convexity is well recognized in the optimization theory and accomplished a significant role in the computational economics, management, decision making, and operation research. Consequently, the generalized convexity and generalized monotonocities are fundamental tools in these areas of research.

Hanson [1] introduced the generalized version of convex function namely invex function. Further, generalized invex monotonocities have been explored by Ruiz-Garzon et al. [2] and Yang et al. [3]. A step forward Yang et al. [4] found that there were some errors in [2] and modified the results of RuizGarzon et al. [2] and they also proposed the notion of strong pseudo invex monotonicity and quasi-invex monotonicity. In addition, Antczak [5, 6] and Suneja et al. [7] deliberated the properties and execution of preinvex functions and their generalizations for the nonlinear programming problems. Aghezzaf and Hachimi [8] presented the differentiable type I functions and derived the appropriate duality results for a Mond-Weir type dual. Gulati et al. [9] presented the more generalized class of convex function, called $(F, \alpha, \rho, d)-V$ type I functions, and derived sufficiency and duality results for a multiobjective programming problem. Recently, Ahmad et al. [10] discussed twice weakly differentiable and interval valued bonvex functions. Duality results are also discussed for Mangasarian type dual model.

The notion of fuzzy generalized convex functions has been investigated by several authors and presently, it is a fascinating and exciting area of research. In 1989, Nanda and Kar [11] presented the idea of convex fuzzy mappings and obtained the convex fuzzy mapping under the epigraph of convex function in a convex set. In [12], Furukawa proposed the concept of convexity and Lipschitz continuity for the category of fuzzy valued functions. Yan and $\mathrm{Xu}$ [13] investigated the convexity and quasi-convexity of fuzzy mapping involving the concept of ordering [14]. These concepts further generalized for fuzzy functions by Noor [15]. Qiu and Zhang [16] discussed the convexity invariance of fuzzy sets under the extension principles. In [17-21], authors proposed the perception of fuzzy convex mappings and presented the idea of invexity, pseudoconvexity, and pseudoinvexity for fuzzy mappings. Recently, Li et al. [22] introduced the fuzzy generalized convex mappings and discussed the properties. The optimality conditions and duality results are also presented under fuzzy weakly univex functions. Some new types of fuzzy starshapedness and their relationships and basic properties are investigated in [23]. Many authors have studied the applications of generalized fuzzy convex mappings to fuzzy optimization problems (see [24-28]). The purpose of the present paper is to develop the notion of generalized invex monotonocities for fuzzy valued mappings. 


\section{Notations and Preliminaries}

Let $U$ be the universal set whose standard element is denoted by $u$. A fuzzy set $\delta$ in $U$ is a function $\delta: U \rightarrow[0,1]$ and support of $\delta$ is denoted by $S(\delta)$ and is given by

$$
S(\delta)=\{u \in U: \delta(u)>0\} .
$$

Definition 1. If $\delta$ be a fuzzy set in $U$ and $\alpha \in[0,1]$, then the $\alpha$ - cut of fuzzy set $\delta$ is defined as

$$
[\delta]^{\alpha}=\{u \in U: \delta(u) \geq \alpha\} .
$$

Definition 2. A fuzzy number $\delta$ is a fuzzy set in $R^{1}$ and satisfies the following conditions:

(i) $\delta$ is normal; that is, there exits $u_{0} \in R$ such that $\delta\left(u_{0}\right)=1$,

(ii) $\delta$ is upper semicontinuous,

(iii) $\delta$ is fuzzy convex that is, $\delta(\lambda u+(1-\lambda) v) \geq$ $\min \{\delta(u), \delta(v)\}, u, v \in R, \lambda \in[0,1]$,

(iv) $[\delta]^{0}$ is compact.

The LR-fuzzy numbers first introduced in [29] are defined as follows:

Definition 3. Let $L, R:[0,1] \rightarrow[0,1]$ be two upper semicontinuous and decreasing functions and $L(0)=R(0)=1$, and $L(1)=R(1)=0$. Then the fuzzy number $\delta$ is given by

$$
\delta(u)= \begin{cases}L\left(\frac{a-u}{\sigma}\right) & a-\sigma \leq u<a, \\ 1 & a \leq u<b, \\ R\left(\frac{u-b}{\beta}\right) & b \leq u<b+\beta, \\ 0 & \text { otherwise }\end{cases}
$$

where $\sigma, \beta>0$ and $a \leq b$.

Let $E$ be the set of fuzzy numbers and let $\delta \in E$ be a fuzzy number if and only if $[\delta]^{\alpha}$ is a nonempty compact convex subset of $R^{1}$. This is denoted by $\left[\delta_{*}(\alpha), \delta^{*}(\alpha)\right]$ for each $\alpha \in[0,1]$. A fuzzy number is resolved by the end points of the interval $\left[\delta_{*}(\alpha), \delta^{*}(\alpha)\right]$.

A real number $u \in R^{1}$ is a particular case of fuzzy number and is specified by

$$
\tilde{u}(t)= \begin{cases}1 & \text { if } t=u \\ 0 & t \neq u\end{cases}
$$

The parametric form of a fuzzy number $\delta$ is presented as

$$
\left\{\left(\delta_{*}(\alpha), \delta^{*}(\alpha), \alpha\right): \alpha \in[0,1]\right\} .
$$

The following is the characterization of a fuzzy number in terms of the end point $\delta_{*}(\alpha)$ and $\delta^{*}(\alpha)$.
Lemma 4 (see [14]). Assume that $\delta_{*}:[0,1] \rightarrow R$ and $\delta^{*}$ : $[0,1] \rightarrow R$ satisfy the following conditions:

(i) $\delta_{*}:[0,1] \rightarrow R$ is bounded increasing function,

(ii) $\delta^{*}:[0,1] \rightarrow R$ is bounded decreasing function,

(iii) $\delta_{*}(1) \leq \delta^{*}(1)$,

(iv) For $0<k \leq 1, \lim _{\alpha \rightarrow k_{-}} \delta_{*}(\alpha)=\delta_{*}(k)$ and $\lim _{\alpha \rightarrow k_{-}} \delta^{*}(\alpha)=\delta^{*}(k)$,

(v) $\lim _{\alpha \rightarrow 0+} \delta_{*}(\alpha)=\delta_{*}(0)$ and $\lim _{\alpha \rightarrow 0+} \delta^{*}(\alpha)=\delta^{*}(0)$.

For $\delta, \gamma \in E, \lambda \in R$, the fuzzy addition and scalar multiplication for $u \in R$ are defined as

$$
\begin{aligned}
(\delta \mp \gamma)(u) & =\sup _{v+w=u} \min [\delta(v), \gamma(w)], \\
(\lambda \delta)(u) & = \begin{cases}\delta\left(\lambda^{-1} u\right), & \lambda \neq 0, \\
0, & \lambda=0 .\end{cases}
\end{aligned}
$$

We know that for any $\delta, \gamma \in E, \lambda \delta \in E,[\delta \mp \gamma]^{\alpha}=[\delta]^{\alpha}+[\gamma]^{\alpha}$ and $[\lambda \delta]^{\alpha}=\lambda[\delta]^{\alpha}$, that is, for each $\alpha \in[0,1]$,

$$
\begin{aligned}
&(\delta \widetilde{+\gamma})_{*}(\alpha)=\delta_{*}(\alpha)+\gamma_{*}(\alpha), \\
&(\delta \widetilde{+\gamma})^{*}(\alpha)=\delta^{*}(\alpha)+\gamma^{*}(\alpha),(\lambda \delta)(\alpha)= \begin{cases}\lambda \delta_{*}(\alpha), & \lambda \geq 0, \\
\lambda \delta^{*}(\alpha), & \lambda<0 .\end{cases} \\
&(\lambda \delta)(\alpha)= \begin{cases}\lambda \delta^{*}(\alpha), & \lambda \geq 0, \\
\lambda \delta_{*}(\alpha), & \lambda<0 .\end{cases}
\end{aligned}
$$

For any $\delta, \gamma \in E, \delta \preceq \gamma$ iffor each $\alpha \in[0,1], \delta_{*}(\alpha) \leq \gamma_{*}(\alpha)$ and $\delta^{*}(\alpha) \leq \gamma^{*}(\alpha)$. If $\delta \preceq \gamma$ and $\gamma \preceq \delta$ then $\delta=\gamma$. We say that $\delta \prec \gamma$, and there exists some $\alpha_{0} \in[0,1]$, such that $\delta_{*}\left(\alpha_{0}\right)<\gamma_{*}\left(\alpha_{0}\right)$ or $\delta^{*}\left(\alpha_{0}\right)<\gamma^{*}\left(\alpha_{0}\right)$. For $\delta, \gamma \in E$, if either $\gamma \preceq \delta$ or $\delta \preceq \gamma$, then $\delta$ and $\gamma$ are comparable, or else they are not comparable, where $\preceq$ is a partial order relation on $E$.

Definition 5 (triangular fuzzy number). A fuzzy number is called a triangular fuzzy number if $\delta_{*}(1)=\delta^{*}(1)$. In addition a fuzzy number $\delta \in E$ is said to be linear, if $\delta_{*}(\alpha)$ and $\delta^{*}(\alpha)$ are linear. A fuzzy triangular number is denoted by $\left\langle\delta_{*}(0), \delta_{*}(1), \delta^{*}(0)\right\rangle$. For the triangular fuzzy number $\delta=$ $\langle 0,1,3\rangle$, we have $[\delta]^{\alpha}=[\alpha, 3-2 \alpha]$.

Definition 6 (fuzzy mapping). Let $U \in R^{n}$ and let $\widetilde{F}$ : $U \rightarrow E$ be a fuzzy mapping. Then the $\alpha$ - cut of $\widetilde{F}(u)$ is given by $\widetilde{F}(u)[\alpha]=\left[F_{*}(u, \alpha), F^{*}(u, \alpha)\right]$, where $F_{*}(u, \alpha)=$ $\min \{\widetilde{F}(u)[\alpha]\}$ and $F^{*}(u, \alpha)=\max \{\widetilde{F}(u)[\alpha]\}$. Thus $\widetilde{F}(u)$ is represented by the two functions $F_{*}(u, \alpha)$ and $F^{*}(u, \alpha)$; these functions are defined from $U \times[0,1]$ to $R$. Moreover, $F_{*}(u, \alpha)$ is bounded increasing function of $\alpha$ and $F^{*}(u, \alpha)$ is bounded decreasing function of $\alpha$ and $F_{*}(u, \alpha) \leq F^{*}(u, \alpha)$ for each $\alpha \in[0,1]$.

Definition 7 (continuity of a fuzzy mapping). If $\widetilde{F}: U \subseteq R^{n} \rightarrow$ $E$ is a fuzzy mapping, then $\widetilde{F}(u)$ is said to be continuous at $u \in U$, if for each $\alpha \in[0,1]$ both the end point functions $F_{*}(u, \alpha)$ and $F^{*}(u, \alpha)$ are continuous at $u$. 
Differentiability of the functions is one of the important tool of the generalized convexity and invex monotonocities. In this paper our aim is to study the concept of fuzzy invex monotone and fuzzy invex function, so we will discuss the notion of differentiability for fuzzy mappings. Puri and Ralescu [30] presented the concept of $\mathrm{H}$-differentiability for fuzzy mappings. Further the concept of S-differentiability, G-differentiability, and weak differentiability was given by Seikkala [31] and Bede and Gal [32], respectively. RufiánLizana et al. [33] pointed that G-differentiability is more general than $\mathrm{H}$-differentiability and S-differentiability for fuzzy mappings. In [34], Bede and Stefanini showed that a weak differentiability of fuzzy mappings is different from G-differentiability. Therefore, it will be worthwhile to study the generalized invex monotonocities and invex functions by using weakly differentiable fuzzy mappings.

Definition 8 (weakly differentiable function [26]). Let $\widetilde{F}$ : $U \subseteq R^{n} \rightarrow E$ be a fuzzy mapping. If the derivatives of $F_{*}(u, \alpha)$, $F^{*}(u, \alpha)$ with respect to $u \in U$ for each $\alpha \in[0,1]$ exist and are denoted by $F_{*}^{\prime}(u, \alpha),{F^{*}}^{\prime}(u, \alpha)$, respectively, then $F(u)$ is said to be weakly differentiable.

\section{Generalized Convexity and Invex Monotonocities}

In this section, we collected some basic definitions of generalized invex functions and generalized invex monotonocities.

Definition 9. A nonempty set $S \subseteq R^{n}$ is said to be invex if there exists a vector valued function $\eta: R^{n} \times R^{n} \rightarrow R^{n}$, such that $v+\lambda \eta(u, v) \in S$ for any $u, v \in S, \lambda \in[0,1]$.

Hanson [1] introduced the generalized version of convex function, namely, invex function. A function $f: R^{n} \rightarrow R$ is said to be invex if there exists a vector valued function $\eta$ : $R^{n} \times R^{n} \rightarrow R$ such that the next inequality

$$
f(v+\lambda \eta(u, v)) \leq \lambda f(u)+(1-\lambda) f(v)
$$

holds, for all $u, v \in R^{n}$.

Definition 10 (pseudoinvex monotone [2]). Let $S \subseteq R^{n}$ be an invex set with respect to $\eta$. Then the function $\phi: S \rightarrow R^{n}$ is said to be (strictly) pseudoinvex monotone on $S$ if

$$
\begin{gathered}
\eta(u, v)^{T} \phi(u) \geq 0 \Longrightarrow \\
\eta(u, v)^{T} \phi(v)(>) \geq 0,
\end{gathered}
$$

for all $u, v \in S$.

Definition 11 (pseudoinvex function [2]). A differentiable function $\phi: S \subseteq R^{n} \rightarrow R^{n}$ is said to be (strictly) pseudoinvex function with respect to $\eta: S \times S \rightarrow R^{n}$ if

$$
\begin{array}{r}
\eta(u, v)^{T} \nabla \phi(u) \geq 0 \Longrightarrow \\
\phi(v)(>) \geq \phi(u),
\end{array}
$$

for all $u, v \in S$.
Definition 12 (pseudoinvex monotone [2]). Let $S \subseteq R^{n}$ be an invex set with respect to $\eta$. Then the function $\phi: S \rightarrow R^{n}$ is said to be (strictly) pseudoinvex monotone on $S$ if

$$
\begin{gathered}
\eta(u, v)^{T} \phi(u) \geq 0 \Longrightarrow \\
\eta(u, v)^{T} \phi(v)(>) \geq 0,
\end{gathered}
$$

for all $u, v \in S$.

Definition 13 (quasi-invex monotone [2]). A function $\phi: S \subseteq$ $R^{n} \rightarrow R^{n}$, defined on an open invex subset $S$ of $R^{n}$, is said to be quasi-invex monotone with respect to $\eta$ on $S$ if

$$
\begin{aligned}
& \eta(u, v)^{T} \phi(u)>0 \Longrightarrow \\
& \eta(u, v)^{T} \phi(v) \geq 0,
\end{aligned}
$$

for all $u, v \in S$.

Definition 14 (quasi-invex function [2]). A function $\phi: S \subseteq$ $R^{n} \rightarrow R^{n}$ which is differentiable on an open invex subset $S$ of $R^{n}$ is said to be quasi-invex function with respect to $\eta$ on $S$ if

$$
\begin{aligned}
\phi(v) & \geq \phi(u) \Longrightarrow \\
\eta(u, v)^{T} \nabla \phi(v) & \leq 0,
\end{aligned}
$$

for all $u, v \in S$.

Now we have the following definitions of strong pseudoinvex monotonicity and strong pseudoinvex functions.

Definition 15 (strong pseudoinvex monotone [3]). A function $\phi: S \subseteq R^{n} \rightarrow R^{n}$ defined on an open invex subset $S$ of $R^{n}$ is said to be strong pseudoinvex monotone with respect to $\eta$ on $S$, if there exists a scalar $m>0$, such that

$$
\begin{aligned}
& \eta(u, v)^{T} \phi(u) \geq 0 \Longrightarrow \\
& \eta(u, v)^{T} \phi(v) \geq m\|\eta(u, v)\|,
\end{aligned}
$$

for all $u, v \in S$.

Definition 16 (strong pseudoinvex function [3]). A function $\phi: S \subseteq R^{n} \rightarrow R^{n}$ which is differentiable on an open invex subset $S$ of $R^{n}$ is said to be strong pseudoinvex function with respect to $\eta$ on $S$ if there exists a scalar $n>0$ such that

$$
\begin{aligned}
\eta(u, v)^{T} \nabla \phi(u) & \geq 0 \Longrightarrow \\
\phi(v) & \geq \phi(u)+n\|\eta(u, v)\|,
\end{aligned}
$$

for all $u, v \in S$.

\section{Weakly Fuzzy Pseudoinvex Monotonicity}

In this section, we propose the new concept of weakly fuzzy pseudoinvex monotone and weakly fuzzy pseudoinvex function and we will prove a necessary condition for weakly fuzzy pseudoinvex monotonicity. 
Definition 17. A fuzzy mapping $\tilde{\phi}: U \rightarrow E$ is said to be (Strictly) weakly fuzzy pseudoinvex monotone with respect to $\eta$ on $U \in R^{n}$ if

$$
\begin{gathered}
\eta(u, v)^{T} \phi_{*}(u, \alpha) \geq 0 \Longrightarrow \\
\eta(u, v)^{T} \phi_{*}(\alpha, v)(>) \geq 0, \\
\eta(u, v)^{T} \phi^{*}(u, \alpha) \geq 0 \Longrightarrow \\
\eta(u, v)^{T} \phi^{*}(\alpha, v)(>) \geq 0,
\end{gathered}
$$

for any $u, v \in U$.

In support of above definition, we have the following example.

Example 18. The triangular fuzzy valued function $\widetilde{\phi}=$ $\langle 2,1,3\rangle u$ is a weakly fuzzy pseudoinvex monotone with respect to $\eta(u, v)=u-v$, where $u \geq v$ and $u, v \geq 0$.

The given function can be written as $\widetilde{\phi}=[(2-\alpha) u,(3-$ $2 \alpha) u]$, where $\phi_{*}(u, \alpha)=(2-\alpha) u$ and $\phi^{*}(u, \alpha)=(3-2 \alpha) u$ and $\eta(v, u)=u-v$, where $u \geq v$ and $u, v \geq 0$. Then it is easy to see that $\widetilde{\phi}(u)$ is a weakly fuzzy monotone.

Definition 19. A fuzzy mapping $\tilde{\psi}: U \rightarrow E$, which is weakly differentiable on $U \in R^{n}$ is said to be (strictly) weakly fuzzy pseudoinvex function with respect to $\eta$ on $U$ if

$$
\begin{aligned}
\eta(u, v)^{T} \nabla \psi_{*}(u, \alpha) & \geq 0 \Longrightarrow \\
\psi_{*}(v, \alpha)(>) & \geq \psi_{*}(u, \alpha), \\
\eta(u, v)^{T} \nabla \psi^{*}(u, \alpha) & \geq 0 \Longrightarrow \\
\psi^{*}(v, \alpha)(>) & \geq \psi^{*}(u, \alpha)
\end{aligned}
$$

for any $u, v \in U$.

Moreover, we have the following example for weakly fuzzy pseudoinvex function.

Example 20. The triangular fuzzy valued function $\tilde{\psi}=$ $\langle 2,1,3\rangle e^{u}$ is a weakly fuzzy pseudoinvex function with respect to $\eta(v, u)=v-u$ and $u \leq v$.

The given function can be written as $\tilde{\psi}=\left[(2-\alpha) e^{u},(3-\right.$ $\left.2 \alpha) e^{u}\right]$; that means $\psi_{*}(u, \alpha)=(2-\alpha) e^{u}, \psi^{*}(u, \alpha)=(3-$ $2 \alpha) e^{u}$, and then by some simple computation we can see the following:

$$
\begin{aligned}
\eta(u, v)^{T} \nabla \psi_{*}(u, \alpha) & \geq 0 \Longrightarrow \\
\psi_{*}(v, \alpha) & \geq \psi_{*}(u, \alpha), \\
\eta(u, v)^{T} \nabla \psi^{*}(u, \alpha) & \geq 0 \Longrightarrow \\
\psi^{*}(v, \alpha) & \geq \psi^{*}(u, \alpha) .
\end{aligned}
$$

Hence $\widetilde{\psi}=\langle 2,1,3\rangle e^{u}$ is a weakly fuzzy pseudoinvex function.
Now we will define the Condition $\mathrm{C}$ as follows.

Condition $C$. For vector valued function $\eta: U \times U \rightarrow R^{n}$ the following equations are called Condition $\mathrm{C}$ :

$$
\begin{aligned}
& \eta(v, v+\lambda \eta(u, v))=-\lambda \eta(u, v), \\
& \eta(u, v+\lambda \eta(u, v))=(1-\lambda) \eta(u, v),
\end{aligned}
$$

for any $u, v \in U$ and $\lambda \in[0,1]$. Moreover, we have the following result for subsequent use.

Note 21 (see [2]). From Condition C, we have

$$
\eta(v+\bar{\lambda} \eta(u, v), v)=\bar{\lambda} \eta(u, v) .
$$

Theorem 22. Let $\tilde{\phi}: U \subseteq R^{n} \rightarrow E$ be a fuzzy mapping on an open invex set $U$ with respect to $\eta$ and $\eta$ satisfies Condition C. Let $\widetilde{\phi}$ be weakly differentiable on $U$ with the following assumptions:

(i) $\phi_{*}(v, \alpha)>\phi_{*}(u, \alpha) \Rightarrow \eta(u, v)^{T} \nabla \phi_{*}(v+\bar{\lambda} \eta(u, v), \alpha)<$ 0 and $\phi^{*}(v, \alpha)>\phi^{*}(u, \alpha) \Rightarrow \eta(u, v)^{T} \nabla \phi^{*}(v+$ $\bar{\lambda} \eta(u, v), \alpha)<0$, for some $\bar{\lambda} \in(0,1)$.

(ii) $\nabla \tilde{\phi}$ is weakly fuzzy pseudoinvex monotone with respect to $\eta$ on $U$.

Then $\tilde{\phi}$ is a weakly fuzzy pseudoinvex function with respect to $\eta$ on $U$.

Proof. Suppose that

$$
\begin{aligned}
& \eta(u, v)^{T} \nabla \phi_{*}(v, \alpha) \geq 0, \\
& \eta(u, v)^{T} \nabla \phi^{*}(v, \alpha) \geq 0 .
\end{aligned}
$$

Our aim is to show that

$$
\begin{aligned}
& \phi_{*}(u, \alpha) \geq \phi_{*}(v, \alpha), \\
& \phi^{*}(u, \alpha) \geq \phi^{*}(v, \alpha) .
\end{aligned}
$$

Assume to the contrary that

$$
\begin{gathered}
\phi_{*}(u, \alpha)<\phi_{*}(v, \alpha), \\
\phi^{*}(u, \alpha)<\phi^{*}(v, \alpha) .
\end{gathered}
$$

By the assumption (i)

$$
\begin{aligned}
& \eta(u, v)^{T} \nabla \phi_{*}(v+\bar{\lambda} \eta(u, v), \alpha)<0, \\
& \eta(u, v)^{T} \nabla \phi^{*}(v+\bar{\lambda} \eta(u, v), \alpha)<0,
\end{aligned}
$$

for some $\bar{\lambda} \in(0,1)$.

By Note 21 and (25), we have the following inequalities:

$$
\begin{aligned}
& \eta(v+\bar{\lambda} \eta(u, v), v)^{T} \nabla \phi_{*}(v+\bar{\lambda} \eta(u, v), \alpha)<0, \\
& \eta(v+\bar{\lambda} \eta(u, v), v)^{T} \nabla \phi^{*}(v+\bar{\lambda} \eta(u, v), \alpha)<0 .
\end{aligned}
$$


By the assumption that $\nabla \tilde{\phi}$ is weakly fuzzy pseudo monotone with respect to $\eta$, then it follows from (26) that

$$
\begin{aligned}
& \eta(v+\bar{\lambda} \eta(u, v), v)^{T} \nabla \phi_{*}(v, \alpha)<0, \\
& \eta(v+\bar{\lambda} \eta(u, v), v)^{T} \nabla \phi^{*}(v, \alpha)<0,
\end{aligned}
$$

where $\bar{\lambda} \in(0,1)$.

Since $\bar{\lambda} \in(0,1)$, then by Note $21,(27)$ become

$$
\begin{aligned}
& \eta(u, v)^{T} \nabla \phi_{*}(v, \alpha)<0, \\
& \eta(u, v)^{T} \nabla \phi^{*}(v, \alpha)<0 .
\end{aligned}
$$

The above inequalities contradict (21). Hence $\widetilde{\phi}$ is a weakly fuzzy pseudoinvex function with respect to $\eta$.

Theorem 23. Let $\tilde{\phi}: U \subseteq R^{n} \rightarrow E$ be a fuzzy mapping on an open invex set $U$ with respect to $\eta$ and $\eta$ satisfies the Condition $C$. Let $\tilde{\phi}$ be weakly differentiable on $U$ with the following assumptions:

(i) $\phi_{*}(v, \alpha) \geq \phi_{*}(u, \alpha) \Rightarrow \eta(u, v)^{T} \nabla \phi_{*}(v+\bar{\lambda} \eta(u, v), \alpha) \leq$ 0 and $\phi^{*}(v, \alpha) \geq \phi^{*}(u, \alpha) \Rightarrow \eta(u, v)^{T} \nabla \phi^{*}(v+$ $\bar{\lambda} \eta(u, v), \alpha) \leq 0$, for each $u, v \in U$ and $\bar{\lambda} \in(0,1)$,

(ii) $\nabla \tilde{\phi}$ is strictly weakly fuzzy pseudoinvex monotone with respect to $\eta$ on $U$.

Then $\tilde{\phi}$ is a strictly weakly fuzzy pseudoinvex function with respect to $\eta$ on $U$.

Proof. Suppose that

$$
\begin{gathered}
\eta(u, v)^{T} \nabla \phi_{*}(v, \alpha) \geq 0, \\
\eta(u, v)^{T} \nabla \phi^{*}(v, \alpha) .
\end{gathered}
$$

Our aim is to show that

$$
\begin{aligned}
& \phi_{*}(u, \alpha)>\phi_{*}(v, \alpha), \\
& \phi^{*}(u, \alpha)>\phi^{*}(v, \alpha) .
\end{aligned}
$$

Assume to the contrary that

$$
\begin{aligned}
& \phi_{*}(u, \alpha) \leq \phi_{*}(v, \alpha), \\
& \phi^{*}(u, \alpha) \leq \phi^{*}(v, \alpha) .
\end{aligned}
$$

By the assumption (i), we have

$$
\begin{aligned}
& \eta(u, v)^{T} \nabla \phi_{*}(v+\bar{\lambda} \eta(u, v), \alpha) \leq 0, \\
& \eta(u, v)^{T} \nabla \phi^{*}(v+\bar{\lambda} \eta(u, v), \alpha) \leq 0,
\end{aligned}
$$

for some $\bar{\lambda} \in(0,1)$.

By Condition $\mathrm{C}$, we have

$$
\eta(v, v+\bar{\lambda} \eta(u, v))=-\bar{\lambda} \eta(u, v)
$$

Utilizing (33) in (32), we have

$$
\begin{aligned}
& \eta(v, v+\bar{\lambda} \eta(u, v))^{T} \nabla \phi_{*}(v+\bar{\lambda} \eta(u, v), \alpha) \geq 0, \\
& \eta(v, v+\bar{\lambda} \eta(u, v))^{T} \nabla \phi^{*}(v+\bar{\lambda} \eta(u, v), \alpha) \geq 0,
\end{aligned}
$$

for some $\bar{\lambda} \in(0,1)$.

By the strictly weakly fuzzy pseudo monotonicity of $\nabla \tilde{\phi}$ with respect to $\eta$ and (34)

$$
\begin{aligned}
& \eta(v, v+\bar{\lambda} \eta(u, v))^{T} \nabla \phi_{*}(v, \alpha)>0, \\
& \eta(v, v+\bar{\lambda} \eta(u, v))^{T} \nabla \phi^{*}(v, \alpha)>0 .
\end{aligned}
$$

Applying the Condition $C$ and the fact that $\bar{\lambda} \in(0,1)$, then (35) takes the form

$$
\begin{aligned}
& \eta(u, v)^{T} \nabla \phi_{*}(v, \alpha)<0, \\
& \eta(u, v)^{T} \nabla \phi^{*}(v, \alpha)<0 .
\end{aligned}
$$

The above inequalities contradict (29). Hence, $\tilde{\phi}$ is a strictly weakly fuzzy pseudoinvex function with respect to $\eta$.

\section{Weakly Fuzzy Quasi-invex Monotonicity}

In this section, necessary conditions for weakly fuzzy quasiinvex monotone are discussed.

Definition 24. A fuzzy mapping $\widetilde{\phi}: U \subseteq R^{n} \rightarrow E$ is said to be weakly fuzzy quasi-invex monotone with respect to $\eta$ on $U \subseteq R^{n}$ if

$$
\begin{aligned}
& \eta(u, v)^{T} \phi_{*}(u, \alpha)>0 \Longrightarrow \\
& \eta(u, v)^{T} \phi_{*}(v, \alpha) \geq 0, \\
& \eta(u, v)^{T} \phi^{*}(u, \alpha)>0 \Longrightarrow \\
& \eta(u, v)^{T} \phi^{*}(v, \alpha) \geq 0,
\end{aligned}
$$

for any $u, v \in U$.

Example 25. The triangular fuzzy valued function $\widetilde{\phi}(u)=$ $\langle 0,1,2\rangle u^{2}$ is a weakly fuzzy quasi-invex monotone with respect to $\eta(u, v)=u-v$, where $u>v$ and $u>0, v \geq 0$.

$\widetilde{\phi}(u)=\langle 0,1,2\rangle u^{2}=\left[\alpha u^{2},(2-\alpha) u^{2}\right]$; we have

$$
\begin{aligned}
& \phi^{*}(u, \alpha)=(2-\alpha) u^{2}, \\
& \phi_{*}(u, \alpha)=\alpha u^{2} .
\end{aligned}
$$

Now we compute the following

$$
\begin{aligned}
& \eta(u, v) \phi^{*}(u, \alpha)=(u-v)(2-\alpha) u^{2}>0, \\
& \eta(u, v) \phi^{*}(v, \alpha)=(u-v)(2-\alpha) v^{2} \geq 0,
\end{aligned}
$$

for all $u>v$ and $u>0, v \geq 0$.

Similarly, we can compute $\eta(u, v) \phi_{*}(u, \alpha)>0$ and $\eta(u, v) \phi_{*}(v, \alpha) \geq 0$. It is easy to see that $\widetilde{\phi}(u)$ is not weakly fuzzy pseudoinvex monotone function. 
Definition 26. A weakly differentiable fuzzy mapping $\widetilde{\psi}: U \subseteq$ $R^{n} \rightarrow E$ is said to be weakly fuzzy quasi-invex function with respect to $\eta$ on $U \subseteq R^{n}$ if

$$
\begin{aligned}
\psi_{*}(v, \alpha) & \geq \psi_{*}(u, \alpha) \Longrightarrow \\
\eta(u, v)^{T} \nabla \psi_{*}(v, \alpha) & \leq 0, \\
\psi^{*}(v, \alpha) & \geq \psi^{*}(u, \alpha) \Longrightarrow \\
\eta(u, v)^{T} \nabla \psi^{*}(v, \alpha) & \leq 0,
\end{aligned}
$$

for any $u, v \in U$.

Example 27. The fuzzy valued function $\widetilde{\psi}(u)=\langle 0,1,2\rangle e^{u}$ is a weakly fuzzy quasi-invex function with respect to $\eta(u, v)=$ $u-v$ and $u \leq v$.

The given function can be written as $\widetilde{\psi}(u)=\langle 0,1,2\rangle e^{u}=$ $\left[\alpha e^{u},(2-\alpha) e^{u}\right]$; we have

$$
\begin{aligned}
\psi^{*}(u, \alpha) & =(2-\alpha) e^{u}, \\
\psi_{*}(u, \alpha) & =\alpha e^{u}, \\
\nabla \psi^{*}(u, \alpha) & =(2-\alpha) e^{u}, \\
\nabla \psi_{*}(u, \alpha) & =\alpha e^{u} .
\end{aligned}
$$

Now, it is easy to show the following

$$
\begin{aligned}
\psi^{*}(u, \alpha) & \geq \psi^{*}(v, \alpha) \Longrightarrow \\
\eta(u, v) \nabla \psi^{*}(v, \alpha) & \leq 0, \\
\psi_{*}(u, \alpha) & \geq \psi_{*}(v, \alpha) \Longrightarrow \\
\eta(u, v) \nabla \psi_{*}(v, \alpha) & \leq 0
\end{aligned}
$$

for all $u \leq v$. Hence, the function $\widetilde{\psi}(u)$ is a weakly fuzzy quasiinvex function, but not weakly pseudoinvex function.

Theorem 28. Let $\tilde{\phi}: U \subseteq R^{n} \rightarrow E$ be a fuzzy mapping on an open invex set $U$ with respect to $\eta$ and $\eta$ satisfying Condition C. Let $\tilde{\phi}$ be weakly differentiable on $U$ with the following conditions

(i) $\phi_{*}(v, \alpha) \geq \phi_{*}(u, \alpha) \Rightarrow \eta(u, v)^{T} \nabla \phi_{*}(v+\bar{\lambda} \eta(u, v), \alpha)<$ 0 and $\phi^{*}(v, \alpha) \geq \phi^{*}(u, \alpha) \Rightarrow \eta(u, v)^{T} \nabla \phi^{*}(v+$ $\bar{\lambda} \eta(u, v), \alpha)<0$, for some $\bar{\lambda} \in(0,1)$.

(ii) $\nabla \widetilde{\phi}$ is weakly fuzzy quasi-invex monotone with respect to $\eta$ on $U$.

Then $\tilde{\phi}$ is a weakly fuzzy quasi-invex function with respect to $\eta$ on $U$.

Proof. Suppose that $\widetilde{\phi}$ is not weakly fuzzy quasi-invex function; then

$$
\begin{aligned}
& \phi_{*}(v, \alpha) \geq \phi_{*}(u, \alpha), \\
& \phi^{*}(\nu, \alpha) \geq \phi_{*}(u, \alpha) .
\end{aligned}
$$

But

$$
\begin{aligned}
& \eta(u, v)^{T} \nabla \phi_{*}(v, \alpha)>0, \\
& \eta(u, v)^{T} \nabla \phi^{*}(v, \alpha)>0 .
\end{aligned}
$$

By the assumption (i) and (43)

$$
\begin{aligned}
& \eta(u, v)^{T} \nabla \phi_{*}(v+\bar{\lambda} \eta(u, v), \alpha)<0, \\
& \eta(u, v)^{T} \nabla \phi^{*}(v+\bar{\lambda} \eta(u, v), \alpha)<0,
\end{aligned}
$$

for some $\bar{\lambda} \in(0,1)$.

By Condition $\mathrm{C}$, we have

$$
\eta(v, v+\bar{\lambda} \eta(u, v))=-\bar{\lambda} \eta(u, v) .
$$

By utilizing (46) in (45), it is easy to see the following

$$
\begin{aligned}
& \eta(v, v+\bar{\lambda} \eta(u, v))^{T} \nabla \phi_{*}(v+\bar{\lambda} \eta(u, v), \alpha)>0, \\
& \eta(v, v+\bar{\lambda} \eta(u, v))^{T} \nabla \phi^{*}(v+\bar{\lambda} \eta(u, v), \alpha)>0,
\end{aligned}
$$

for some $\bar{\lambda} \in(0,1)$.

Since $\nabla \widetilde{\phi}$ is weakly fuzzy quasi-invex monotone, then from (47) we have

$$
\begin{gathered}
\eta(v, v+\bar{\lambda} \eta(u, v))^{T} \nabla \phi_{*}(v, \alpha) \geq 0, \\
\eta(v, v+\bar{\lambda} \eta(u, v))^{T} \nabla \phi^{*}(v, \alpha) \geq 0 .
\end{gathered}
$$

Again applying the Condition $\mathrm{C}$ and the fact $\bar{\lambda} \in(0,1)$, the above equation reduced to

$$
\begin{aligned}
& \eta(u, v)^{T} \nabla \phi_{*}(v, \alpha) \leq 0, \\
& \eta(u, v)^{T} \nabla \phi^{*}(v, \alpha) \leq 0,
\end{aligned}
$$

which contradicts (44). Hence $\widetilde{\phi}$ is a weakly fuzzy quasi-invex function with respect to $\eta$.

\section{Fuzzy Strong Pseudo Invex Monotonicity}

In this section, we propose the idea of fuzzy strong pseudoinvex monotonicity and fuzzy strong pseudo invex functions. Finally, we prove a necessary condition for fuzzy strong pseudoinvex monotone.

Definition 29. A fuzzy mapping $\widetilde{\phi}: U \subseteq R^{n} \rightarrow E$ is said to be fuzzy strong pseudoinvex monotone with respect to $\eta$ on an invex set $U \subseteq R^{n}$ if there exists a scalar $m$ such that

$$
\begin{aligned}
& \eta(v, u)^{T} \phi_{*}(u, \alpha) \geq 0 \Longrightarrow \\
& \eta(u, v)^{T} \phi^{*}(v, \alpha) \geq m\|\eta(v, u)\|, \\
& \eta(v, u)^{T} \phi^{*}(u, \alpha) \geq 0 \Longrightarrow \\
& \eta(v, u)^{T} \phi^{*}(v, \alpha) \geq m\|\eta(v, u)\|,
\end{aligned}
$$

for any $u, v \in U$. 
Now we have the following example.

Example 30. The triangular fuzzy valued function $\tilde{\phi}=$ $\langle 0,1,4\rangle u^{2}$ is a fuzzy strong pseudoinvex monotone with respect to $\eta(v, u)=u-v$ and $m \leq 0$, where $u \geq v$.

For $\widetilde{\phi}=\langle 0,1,4\rangle u^{2}=\left[\alpha u^{2},(4-3 \alpha) u^{2}\right]$, then we have $\phi_{*}(u, \alpha)=\alpha u^{2}$ and $\phi^{*}(u, \alpha)=(4-3 \alpha) u^{2}$. Now we compute the following

$$
\begin{aligned}
& \eta(v, u)^{T} \phi_{*}(u, \alpha)=(u-v) \alpha u^{2} \geq 0, \\
& \eta(v, u)^{T} \phi_{*}(v, \alpha)=(u-v) \alpha v^{2} \geq m\|u-v\|,
\end{aligned}
$$

for all $m \leq 0$.

Similarly, for $\phi^{*}(u, \alpha)=(4-3 \alpha) u^{2}$, we can show the following

$$
\begin{aligned}
& \eta(v, u)^{T} \phi^{*}(u, \alpha) \geq 0 \Longrightarrow \\
& \eta(v, u)^{T} \phi^{*}(v, \alpha) \geq m\|\eta(v, u)\|,
\end{aligned}
$$

for all $u \geq v$ and $m \leq 0$, but $\widetilde{\phi}(u)$ is neither weakly fuzzy pseudoinvex monotone function nor weakly fuzzy quasiinvex monotone function.

Definition 31. A weakly differentiable fuzzy mapping $\widetilde{\phi}: U \subseteq$ $R^{n} \rightarrow E$ is said to be fuzzy strong pseudoinvex function with respect to $\eta$ on an invex set $U \subseteq R^{n}$ if there exists a scalar $\eta$ such that

$$
\begin{aligned}
\eta(v, u)^{T} \nabla \phi_{*}(u, \alpha) & \geq 0 \Longrightarrow \\
\phi_{*}(v, \alpha) & \geq \phi_{*}(u, \alpha)+n\|\eta(v, u)\|, \\
\eta(v, u)^{T} \nabla \phi^{*}(u, \alpha) & \geq 0 \Longrightarrow \\
\phi^{*}(v, \alpha) & \geq \phi^{*}(u, \alpha)+n\|\eta(v, u)\|,
\end{aligned}
$$

for any $u, v \in U$.

Example 32. The triangular fuzzy valued function $\widetilde{\psi}(u)=$ $\langle 0,1,4\rangle u$ is a fuzzy strong pseudoinvex function with respect to $\eta(v, u)=v-u$ and $n \leq 0$, where $u \leq v$.

We can write $\widetilde{\psi}(u)=\langle 0,1,4\rangle u=[\alpha u,(4-3 \alpha) u]$, and then we have $\psi_{*}(u, \alpha)=\alpha u$ and $\psi^{*}(u, \alpha)=(4-3 \alpha) u$. $\eta(v, u)=$ $v-u \nabla \psi_{*}(u, \alpha)=\alpha$. Then we have

$$
\begin{aligned}
\eta(v, u) \nabla \psi_{*}(u, \alpha) & =(v-u) \alpha \geq 0, \\
\alpha v & \geq \alpha u+n\|v-u\|,
\end{aligned}
$$

for all $u \leq v$ and $n \leq 0$; that means

$$
\psi_{*}(v, \alpha) \geq \psi_{*}(u, \alpha)+n\|\eta(v, u)\| .
$$

Similarly, it is easy to show that

$$
\begin{aligned}
\eta(v, u) \nabla \psi^{*}(u, \alpha) & \geq 0 \Longrightarrow \\
\phi^{*}(v, \alpha) & \geq \phi^{*}(u, \alpha)+n\|\eta(v, u)\| .
\end{aligned}
$$

Hence the function $\widetilde{\psi}(u)=\langle 0,1,4\rangle u$ is a fuzzy strong pseudoinvex function with respect to $\eta(v, u)=v-u$ and $n \leq 0$, where $u \leq v$. It is easy see that $\widetilde{\phi}(u)$ is neither weakly fuzzy pseudoinvex function nor weakly fuzzy quasi-invex function.
Theorem 33. Let $\tilde{\phi}: U \subseteq R^{n} \rightarrow E$ be a fuzzy mapping on an open invex set with respect to $\eta$ and $\eta$ satisfying the Condition C. Let $\tilde{\phi}$ is weakly differentiable on $U$ with the following conditions

(i) $\phi_{*}(u+\eta(v, u), \alpha) \leq \phi_{*}(v, \alpha)$ and $\phi^{*}(u+\eta(v, u), \alpha) \leq$ $\phi^{*}(v, \alpha)$, for all $u, v \in U$.

(ii) $\nabla \widetilde{\phi}$ is a fuzzy strong pseudoinvex monotone with respect to $\eta$ on $U$.

Then $\tilde{\phi}$ is a fuzzy pseudoinvex function with respect to $\eta$ on $U$.

Proof. Suppose

$$
\begin{aligned}
& \eta(v, u)^{T} \nabla \phi_{*}(u, \alpha) \geq 0, \\
& \eta(v, u)^{T} \nabla \phi^{*}(u, \alpha) \geq 0,
\end{aligned}
$$

for any $u, v \in U$.

From the assumption that $U$ is open invex set with respect to $\eta$ and $\eta$ satisfying the Condition $C$, the above inequalities become

$$
\begin{aligned}
& \eta(u+\lambda \eta(v, u), u)^{T} \nabla \phi_{*}(u, \alpha) \geq 0, \\
& \eta(u+\lambda \eta(v, u), u)^{T} \nabla \phi^{*}(u, \alpha) \geq 0 .
\end{aligned}
$$

Since $\nabla \tilde{\phi}$ is a fuzzy strong pseudoinvex monotone with respect to $\eta$, then there exists $m>0$ such that

$$
\begin{gathered}
\eta(u+\lambda \eta(v, u), u)^{T} \nabla \phi_{*}(u+\lambda \eta(v, u), \alpha) \\
\geq m\|\eta(u+\lambda \eta(v, u), u)\|, \\
\eta(u+\lambda \eta(v, u), u)^{T} \nabla \phi^{*}(u+\lambda \eta(v, u), \alpha) \\
\geq m\|\eta(u+\lambda \eta(v, u), u)\| .
\end{gathered}
$$

Again by Condition $\mathrm{C}$ and $\lambda \in(0,1]$ the above inequalities take the form

$$
\begin{aligned}
& \eta(v, u)^{T} \nabla \phi_{*}(u+\lambda \eta(v, u), \alpha) \geq m\|\eta(v, u)\|, \\
& \eta(v, u)^{T} \nabla \phi^{*}(u+\lambda \eta(v, u), \alpha) \geq m\|\eta(v, u)\|,
\end{aligned}
$$

for all $\lambda \in(0,1]$.

Let $\psi_{*}(\lambda)=\phi_{*}(u+\lambda \eta(\nu, u), \alpha)$ and $\psi^{*}(\lambda)=\phi^{*}(u+$ $\lambda \eta(v, u), \alpha)$.

Then from (60) and (61) we have,

$$
\begin{gathered}
\psi_{*}^{\prime}(\lambda) \geq m\|\eta(v, u)\|, \\
\psi^{* \prime}(\lambda) \geq m\|\eta(v, u)\| .
\end{gathered}
$$

Integrating from 0 to 1

$$
\begin{gathered}
\int_{0}^{1} \psi_{*}^{\prime}(\lambda) d \lambda \geq m\|\eta(v, u)\| \int_{0}^{1} 1 d \lambda, \\
\int_{0}^{1} \psi^{* \prime}(\lambda) d \lambda \geq m\|\eta(v, u)\| \int_{0}^{1} 1 d \lambda .
\end{gathered}
$$


After integration, we have the following

$$
\begin{aligned}
& \psi_{*}(1)-\psi_{*}(0) \geq m\|\eta(v, u)\|, \\
& \psi^{*}(1)-\psi^{*}(0) \geq m\|\eta(v, u)\|,
\end{aligned}
$$

or

$$
\begin{gathered}
\phi_{*}(u+\eta(v, u), \alpha)-\phi_{*}(u, \alpha) \geq m\|\eta(v, u)\|, \\
\phi^{*}(u+\eta(v, u), \alpha)-\phi^{*}(u, \alpha) \geq m\|\eta(v, u)\| .
\end{gathered}
$$

By the assumption (i), we acquire

$$
\begin{aligned}
& \phi_{*}(v, \alpha)-\phi_{*}(u, \alpha) \geq m\|\eta(v, u)\|, \\
& \phi^{*}(v, \alpha)-\phi^{*}(u, \alpha) \geq m\|\eta(v, u)\| .
\end{aligned}
$$

Hence, $\tilde{\phi}$ is a fuzzy strong pseudo invex function.

\section{Conclusion}

The concept of fuzzy optimization is well recognized in the literature and many authors are showing their interest in this direction. The idea of fuzzy convexity has been studied by several authors. The motive of the present paper is to project the concept of generalized weakly fuzzy monotonocities and generalized weakly fuzzy invex functions with nontrivial examples. Moreover, we tried to build up the relationship between generalized fuzzy monotones and generalized fuzzy invex functions. The results proved in the present paper generalize the existing results appearing in the literature. Findings of this paper can be used for fuzzy multiobjective programming problems.

\section{Conflicts of Interest}

The authors declare that they have no conflicts of interest.

\section{Authors' Contributions}

All the authors contributed equally to the writing of this paper and approved the final manuscript.

\section{Acknowledgments}

The research of the second author is financially supported by King Fahd University of Petroleum and Minerals, Saudi Arabia, under the Internal Research Project no. IN161058.

\section{References}

[1] M. A. Hanson, "On sufficiency of the Kuhn-Tucker conditions," Journal of Mathematical Analysis and Applications, vol. 80, no. 2, pp. 545-550, 1981.

[2] G. Ruiz-Garzon, R. Osuna-Gomez, and A. R. Lizana, "Generalized invex monotonicity," European Journal of Operational Research, vol. 144, no. 3, pp. 501-512, 2003.

[3] X. M. Yang, X. Q. Yang, and K. L. Teo, "Generalized invexity and generalized invariant monotonicity," Journal of Optimization Theory and Applications, vol. 117, no. 3, pp. 607-625, 2003.
[4] X. M. Yang, X. Q. Yang, and K. L. Teo, "Criteria for generalized invex monotonicities," European Journal of Operational Research, vol. 164, no. 1, pp. 115-119, 2005.

[5] T. Antczak, “( $p, r)$-invex sets and functions," Journal of Mathematical Analysis and Applications, vol. 263, no. 2, pp. 355-379, 2001.

[6] T. Antczak, "Relationships between pre-invex concepts," Nonlinear Analysis. Theory, Methods \& Applications. An International Multidisciplinary Journal, vol. 60, no. 2, pp. 349-367, 2005.

[7] S. K. Suneja, C. Singh, and C. R. Bector, "Generalization of preinvex and b-vex functions," Journal of Optimization Theory and Applications, vol. 76, no. 3, pp. 577-587, 1993.

[8] B. Aghezzaf and M. Hachimi, "Generalized invexity and duality in multiobjective programming problems," Journal of Global Optimization, vol. 18, no. 1, pp. 91-101, 2000.

[9] T. R. Gulati, I. Ahmad, and D. Agarwal, "Sufficiency and duality in multiobjective programming under generalized type I functions," Journal of Optimization Theory and Applications, vol. 135, no. 3, pp. 411-427, 2007.

[10] I. Ahmad, D. Singh, B. A. Dar, and S. Al-Homidan, "On interval valued functions and Magasarian type duality involving Hukuhara derivative," Journal of Computational Analysis and Applications, vol. 21, no. 5, pp. 881-896, 2016.

[11] S. Nanda and K. Kar, "Convex fuzzy mappings," Fuzzy Sets and Systems, vol. 48, no. 1, pp. 129-132, 1992.

[12] N. Furukawa, "Convexity and local Lipschitz continuity of fuzzy-valued mappings," Fuzzy Sets and Systems, vol. 93, no. 1, pp. 113-119, 1998.

[13] H. Yan and J. Xu, "A class of convex fuzzy mappings," Fuzzy Sets and Systems, vol. 129, no. 1, pp. 47-56, 2002.

[14] R. Goetschel Jr. and W. Voxman, "Elementary fuzzy calculus," Fuzzy Sets and Systems, vol. 18, no. 1, pp. 31-43, 1986.

[15] M. A. Noor, "Fuzzy preinvex functions," Fuzzy Sets and Systems, vol. 64, no. 1, pp. 95-104, 1994.

[16] D. Qiu and W. Zhang, "Convexity invariance of fuzzy sets under the extension principles," journal of function spaces and applications, Article ID 849104, 13 pages, 2012.

[17] J. Li and M. A. Noor, "On characterizations of preinvex fuzzy mappings," Computers \& Mathematics with Applications. An International Journal, vol. 59, no. 2, pp. 933-940, 2010.

[18] Y.-R. Syau, "On convex and concave fuzzy mappings," Fuzzy Sets and Systems, vol. 103, no. 1, pp. 163-168, 1999.

[19] Y.-R. Syau, "Invex and generalized convex fuzzy mappings," Fuzzy Sets and Systems, vol. 115, no. 3, pp. 455-461, 2000.

[20] D. Qiu, C. Lu, W. Zhang, and Y. Lan, "Algebraic properties and topological properties of the quotient space of fuzzy numbers based on Mareš equivalence relation," Fuzzy Sets and Systems, vol. 245, pp. 63-82, 2014.

[21] D. Qiu, L. Shu, and Z.-W. Mo, "Notes on fuzzy complex analysis," Fuzzy Sets and Systems, vol. 160, no. 11, pp. 1578-1589, 2009.

[22] L. Li, S. Liu, and J. Zhang, "On fuzzy generalized convex mappings and optimality conditions for fuzzy weakly univex mappings," Fuzzy Sets and Systems, vol. 280, pp. 107-132, 2015.

[23] D. Qiu, L. Shu, and Z.-W. Mo, “On starshaped fuzzy sets," Fuzzy Sets and Systems, vol. 160, no. 11, pp. 1563-1577, 2009.

[24] G. Wang and C. Wu, "Directional derivatives and subdifferential of convex fuzzy mappings and application in convex fuzzy programming," Fuzzy Sets and Systems, vol. 138, no. 3, pp. 559591, 2003. 
[25] H.-C. Wu, “The Karush-KUHn-Tucker optimality conditions in multiobjective programming problems with interval-valued objective functions," European Journal of Operational Research, vol. 196, no. 1, pp. 49-60, 2009.

[26] J. Ramik and M. Vlach, Generalized Concavity in Fuzzy Optimization and Decision Analysis, Kluwer Academics Publishers, Dordrecht, Netherlands, 2002.

[27] Y. Chalco-Cano, W. A. Lodwick, and A. Rufian-Lizana, "Optimality conditions of type KKT for optimization problem with interval-valued objective function via generalized derivative," Fuzzy Optimization and Decision Making. A Journal of Modeling and Computation Under Uncertainty, vol. 12, no. 3, pp. 305-322, 2013.

[28] D. Qiu, F. Yang, and L. Shu, "On convex fuzzy processes and their generalizations," International Journal of Fuzzy Systems, vol. 12, no. 3, pp. 267-272, 2010.

[29] D. Dubois and H. Prade, "Operations on fuzzy numbers," International Journal of Systems Science, vol. 9, no. 6, pp. 613626, 1978.

[30] M. L. Puri and D. A. Ralescu, "Differentials of fuzzy functions," Journal of Mathematical Analysis and Applications, vol. 91, no. 2, pp. 552-558, 1983.

[31] S. Seikkala, "On the fuzzy initial value problem," Fuzzy Sets and Systems, vol. 24, no. 3, pp. 319-330, 1987.

[32] B. Bede and S. G. Gal, "Generalizations of the differentiability of fuzzy-number-valued functions with applications to fuzzy differential equations," Fuzzy Sets and Systems, vol. 151, no. 3, pp. 581-599, 2005.

[33] A. Rufián-Lizana, Y. Chalco-Cano, R. Osuna-Gómez, and G. Ruiz-Garzón, "On invex fuzzy mappings and fuzzy variationallike inequalities," Fuzzy Sets and Systems, vol. 200, pp. 84-98, 2012.

[34] B. Bede and L. Stefanini, "Generalized differentiability of fuzzyvalued functions," Fuzzy Sets and Systems, vol. 230, pp. 119-141, 2013. 


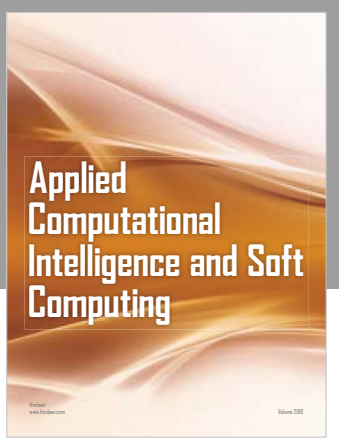

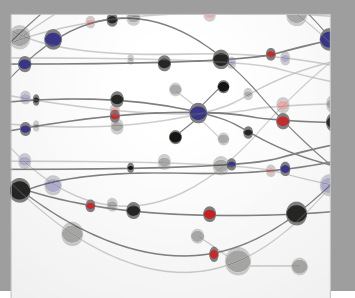

The Scientific World Journal
Submit your manuscripts at

Computing
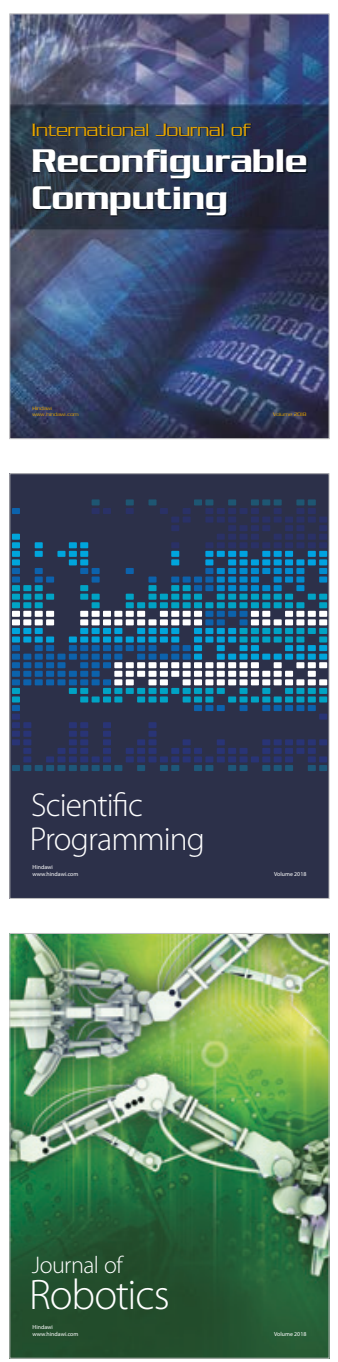

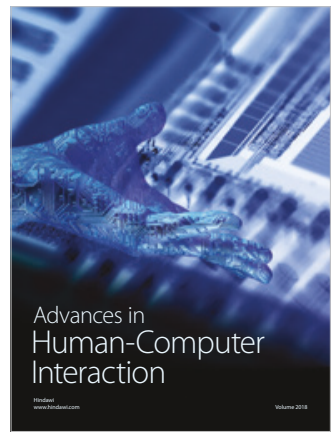

Human-Compute

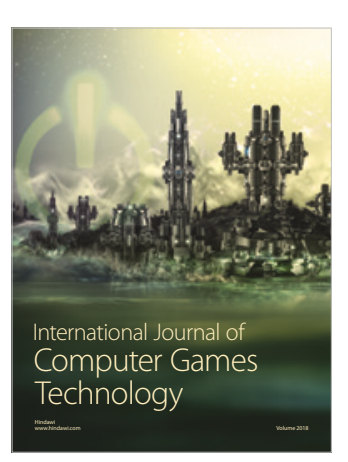

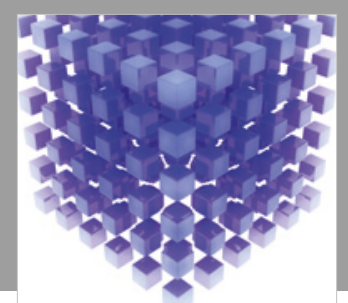

Mathematical Problems in Engineering

\section{Engincering}
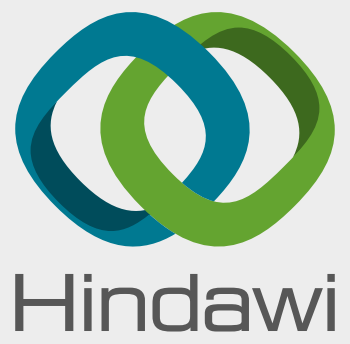

www.hindawi.com
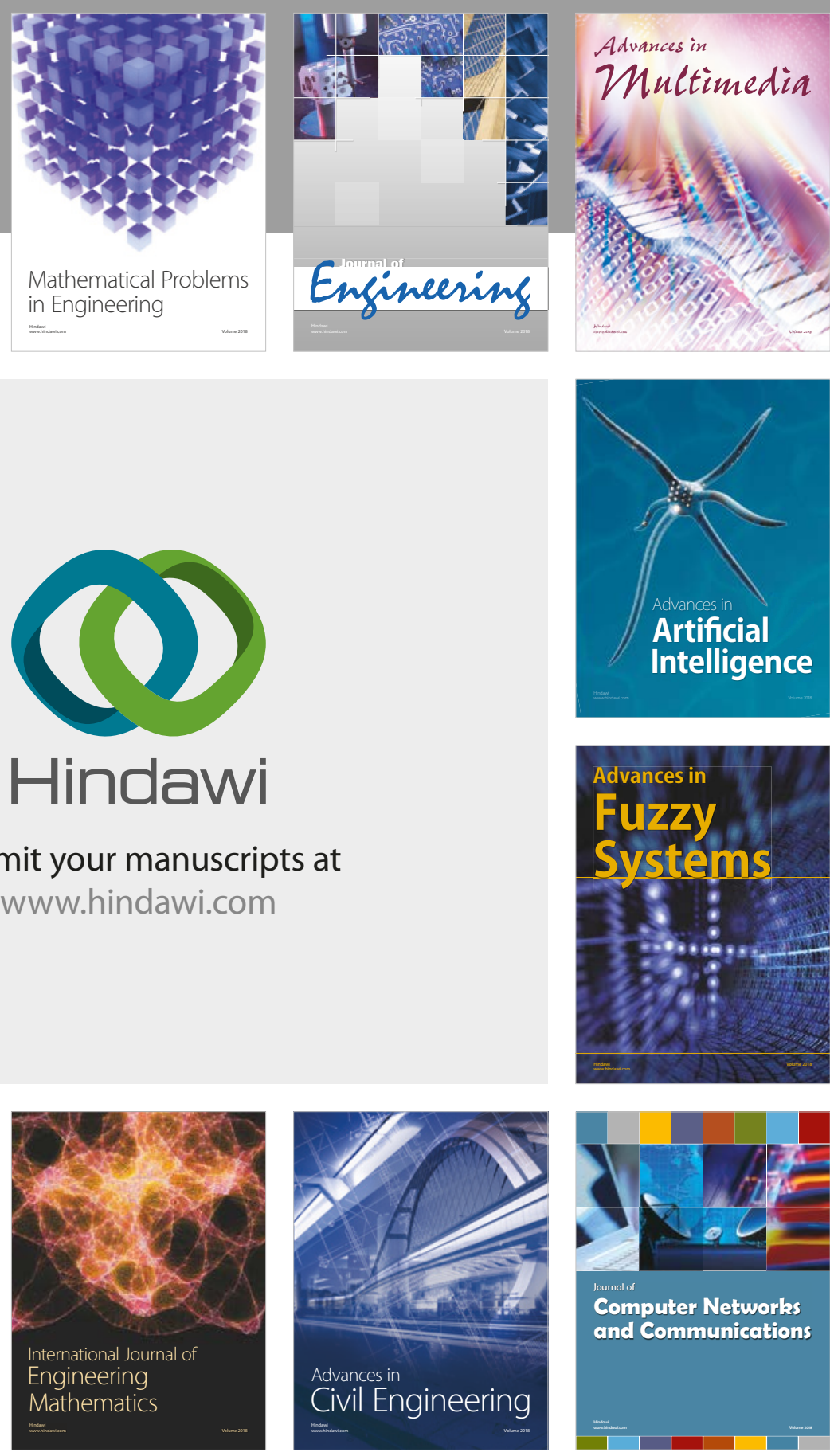

Computer Networks and Communications

Multimedia
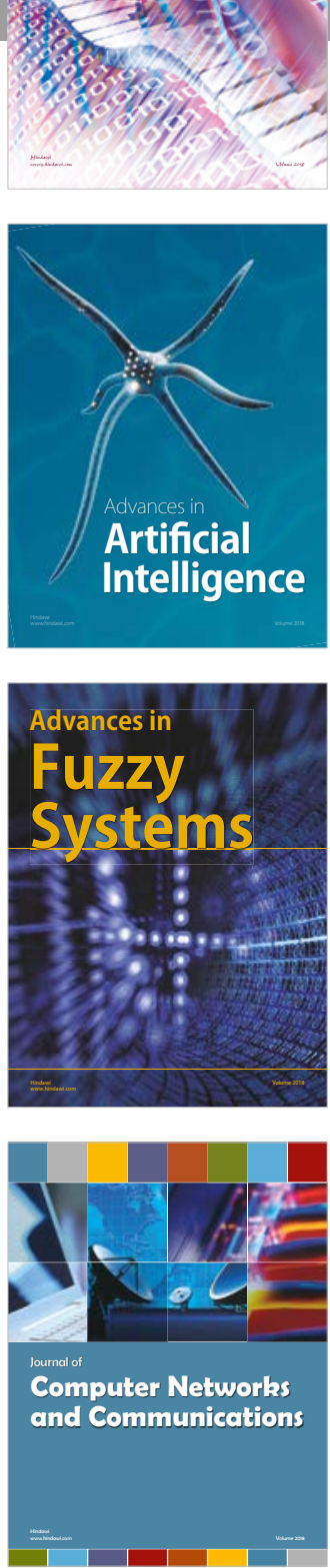

Advances in

Modelling \&

Simulation

in Engineering

interaction

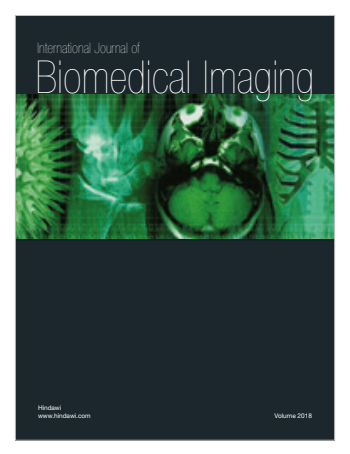

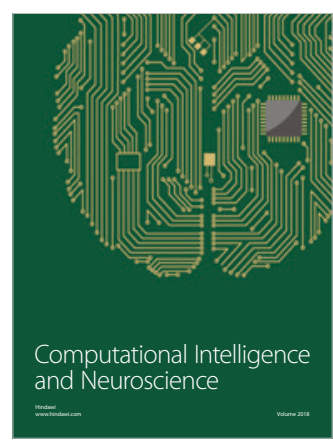

